\title{
ADVANCES IN FLUORESCENCE SPECTROSCOPY: MULTI-PHOTON EXCITATION, ENGINEERED PROTEINS, MODULATION SENSING AND MICROSECOND RHENIUM METAL-LIGAND COMPLEXES*
}

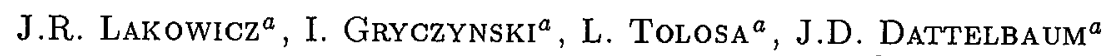
F.N. Castellano ${ }^{a}, L \cdot$ LI $^{a}$ and G. Rao ${ }^{b}$

${ }^{a}$ University of Maryland, School of Medicine 725 West Lombard Street, Baltimore, Maryland 21201, USA

${ }^{b}$ Medical Biotechnology Center, Department of Chemical and Biochemical Engineering 725 West Lombard Street, Baltimore, Maryland 21201, USA

The technology and applications of fluorescence spectroscopy are rapidly advancing. In this overview presentation we summarize some recent developments from this laboratory. Two and three-photon excitation have been observed for a wide variety of intrinsic and extrinsic fluorophores, including tryptophan, tyrosine, DNA stains, membrane probes, and even alkanes. It has been possible to observe multi-photon excitation of biopolymers without obvious photochemical or photo-thermal effects. Although not described in our lecture, another area of increasing interest is the use of engineered proteins for chemical and clinical sensing. We show results for the glucose-galactose binding protein from $E$. coli. The labeled protein shows spectral changes in response to micromolar concentrations of glucose. This protein was used with a novel sensing method based on the modulated emission of the labeled proteins and a long lifetime reference fluorophore. And finally, we describe a recently developed rhenium complex which displays a lifetime near $3 \mu$ s in oxygenated aqueous solution. Such long lifetime probes allow detection of microsecond dynamic processes, bypassing the usual nanosecond timescale limit of fluorescence. The result of these developments in protein engineering, sensing methods, and metal-ligand probe chemistry will be the increased use of fluorescence in clinical chemistry and point-of-care analyses.

PACS numbers: $34.50 . \mathrm{Gb}, 87.64 .-\mathrm{t}, 87.64 . \mathrm{Ni}$

*This article is a Keynote Lecture for the Panel Discussion II "Emerging Topics in Fluorescence Spectroscopy: Multi-Photon Excitation, Long Lifetime Anisotropy Probes and Optical Control of the Excited States" moderated by Professor Joseph R. Lakowicz (editors' note). 


\section{Multi-photon excitation}

Fluorescence spectroscopy usually relies on the interaction of a single photon with the absorbing molecule. However, it has been known since 1931 that molecules can simultaneously interact with two photons. Two-photon absorption was predicted theoretically by Maria Göppert-Mayer in 1931 [1]. This phenomenon only occurs at high light intensities, and was not studied experimentally until after the appearance of lasers in the 1960s and 1970s. The increasing availability of ps and fs lasers has resulted in expanded interest in multi-photon excitation of fluorescence. Since then, two-photon spectroscopy has been used to study the excited state symmetry of organic chromophores [2-4]. Two-photon excitation has been applied to study hydrocarbons [5, 6], porphyrins [7], polyenes [8, 9], protein bound chromophores [10] and indole derivatives [11, 12]. Two-photon excitation has also become a tool in analytical chemistry $[13,14]$. In earlier studies the main emphasis of two-photon spectroscopy was to study the symmetry properties of electronic states [15]. This emphasis was due to selection rules for electronic transitions, which are different for one-photon and two-photon absorption. In recent years, two-photon excitation has been applied to study time dependent intensities and anisotropies for a wide range of fluorophores including membranes labeled with DPH [16], labeled DNA [17, 18], proteins [19], fluorescence standards [20-22], dioxane [23], and alkanes [24-27].

Multi-photon excitation has also become an important tool in fluorescence microscopy and cellular imaging. In two-photon excitation the intensity of the fluorescence depends on the square of the local optical power. Consequently, the excitation is localized to the focal point. Conditions equivalent to confocal microscopy can be achieved without the complexity associated with confocal systems [28-31].

In this laboratory, we have shown that higher order excitation is possible. Using the femtosecond ( $f s$ ) pulses from a Ti:sapphire laser, we have observed simultaneous three-photon excitation of a variety of biochemical fluorophores. This includes probes [32], tryptophan [33, 34], stained DNA [35], and DPH-labeled membranes [36]. Localization of the excitation for a three-photon process can be improved as compared to two-photon excitation [37]. In the following paragraphs, we present recent results for three-photon excitation of tyrosine [38].

We examined the neutral derivative of tyrosine, $N$-acetyl-L-tyrosinamide (NATyrA). The solvent was glycerol-water $(30: 70, \mathrm{~V}: \mathrm{V})$ at $5^{\circ} \mathrm{C}$. These conditions were chosen so the rotational correlation time of NATyrA was comparable to the lifetime, allowing us to determine the time-zero anisotropy from the frequency-domain anisotropy decay. Emission spectra of NATyrA are shown in Fig. 1 for excitation at 280 and $840 \mathrm{~nm}$. The emission spectra are essentially identical, indicating that the emission arises from the same lowest singlet state with 280 and $840 \mathrm{~nm}$ excitation. Since NATyrA does not absorb above $300 \mathrm{~nm}$, it seemed unlikely that two-photon excitation could occur with wavelengths above $600 \mathrm{~nm}$. To determine the mode of excitation at $840 \mathrm{~nm}$, we examined the dependence of the emission intensity on laser power (Fig. 2). We found the dependence characteristic of three-photon excitation. Similar results were obtained for excitation from 780 to $855 \mathrm{~nm}$ (not shown). 

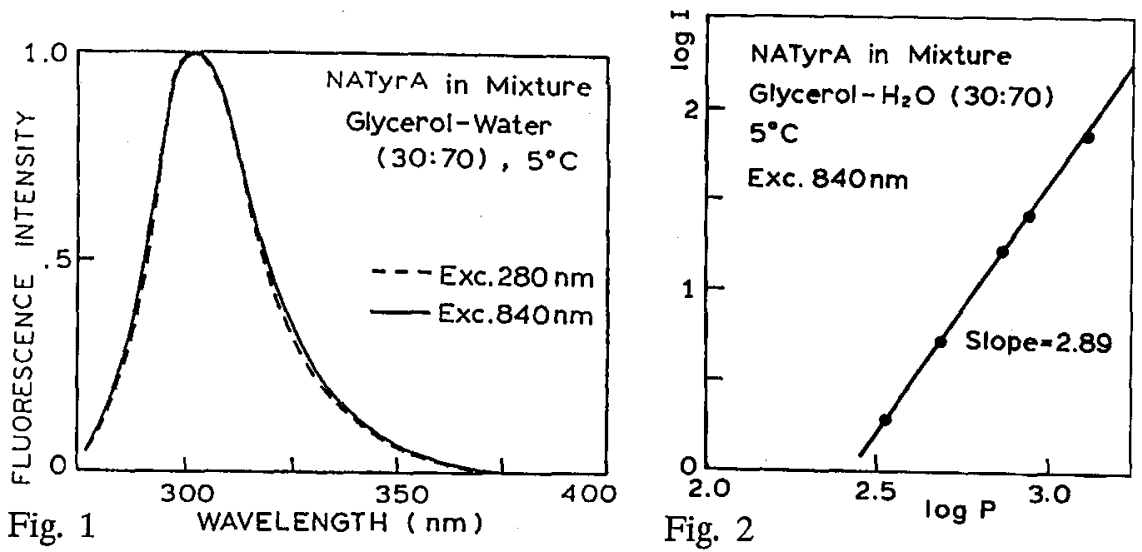

Fig. 1. Fluorescence spectra of NATyrA in a glycerol-water $(30: 70)$ mixture at $5^{\circ} \mathrm{C}$ for excitation at $280 \mathrm{~nm}$ (one-photon) and $840 \mathrm{~nm}$ (three-photon).

Fig. 2 Dependence of the emission intensity $(I)$ of NATyrA on laser power $(P)$.

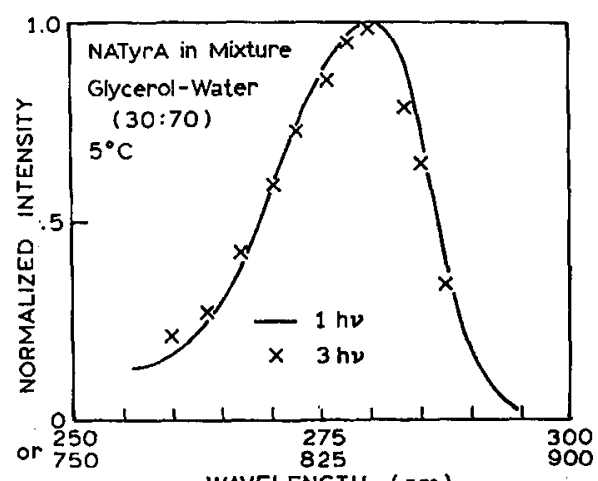

Fig. 3

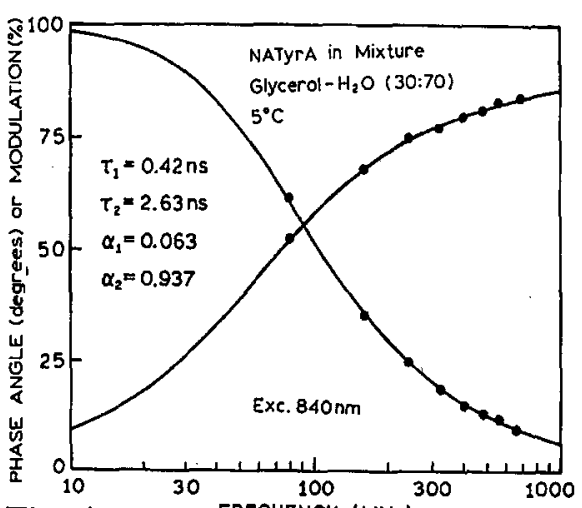

Fig. 4

Fig. 3. Fluorescence excitation spectra for one-photon (-) and three-photon absorption. To determine the three-photon excitation, the fluorescence signal $(I)$ was divided by the cube of the laser power $(P)$ and then normalized.

Fig. 4. Frequency-domain intensity decay of NATyrA in glycerol-water (30:70) mixture at $5^{\circ} \mathrm{C}$ with three-photon excitation.

It is known that the absorption spectra can be different for one- and two-photon excitation. To be more precise, the two-photon absorption spectrum need not look like the one-photon spectrum shifted to twice the wavelength. Hence, we examined the three-photon excitation spectrum of NATyrA (Fig. 3). The results indicate that the excitation spectra are essentially identical. We note that it is difficult to accurately measure multi-photon excitation spectra because the local power depends on the details of the optical focusing and on the mode of the laser. The mode can change with time or can depend on the chosen wavelength. Hence, small changes in the spectra may be present but not observable with the present degree of precision. 
We also examined the intensity decay of NATyrA with one- and three-photon excitation (Fig. 4). Similar intensity decays were observed for one-photon (not shown) and three-photon excitation. This indicates the same excited state for one- and three-photon induced fluorescence. The intensity decays do not depend on excitation wavelength. The same intensity decays were observed for $780 \mathrm{~nm}$, $840 \mathrm{~nm}$, or $855 \mathrm{~nm}$ excitation.

We attempted to measure the fundamental anisotropy of tyrosine in frozen solution. Unfortunately, we found the signal was unstable under the conditions needed for three-photon excitation. Hence, we measured the frequency-domain anisotropy decay (Fig. 5). These frequency-domain data were fit simultaneously to yield one global correlation time $\Theta$ and five non-global zero-time anisotropies $r_{03}(\lambda)$ (Fig. 5 and Table I). The recovered correlation time of $251 \pm 4$ ps is about 4 -fold longer than in aqueous solution [39] due to the higher viscosity. The zero-time anisotropies were dependent on excitation wavelength and ranged from 0.256 for $855 \mathrm{~nm}$ to 0.119 for $780 \mathrm{~nm}$. The wavelength dependent time-zero anisotropies represent anisotropy spectrum of NATyrA (Fig. 6).
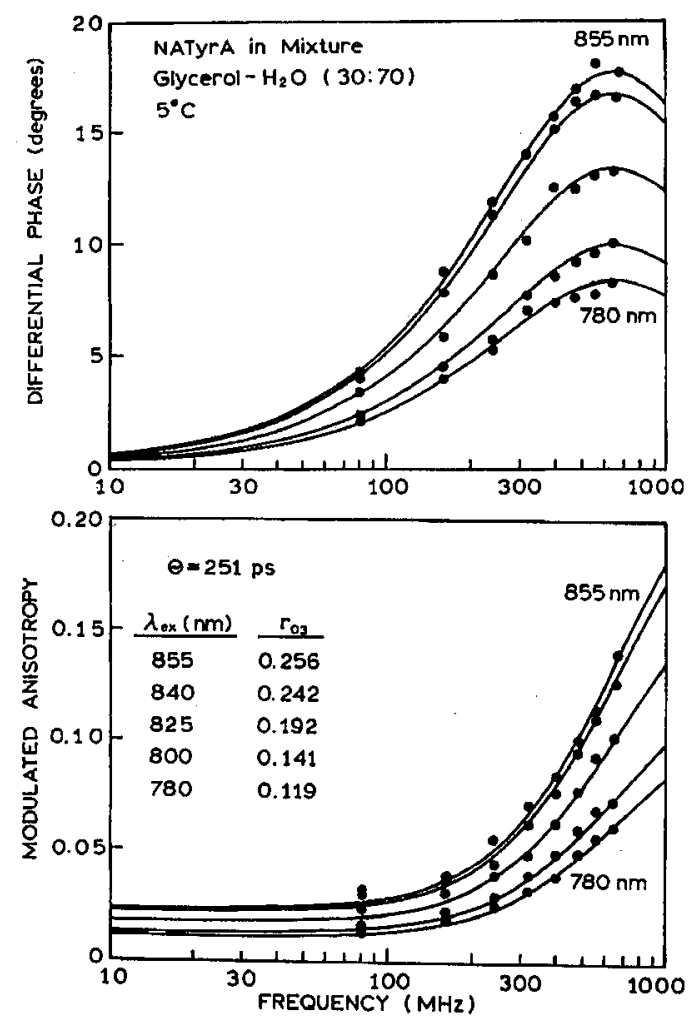

Fig. 5. Frequency-domain anisotropy decays of NATyrA for three-photon excitation from 780 to $855 \mathrm{~nm}(\bullet)$. The lines are the global fit to the data. 
TABLE I

Anisotropy decays of $N$-acetyl-L-tyrosinamide with one and three-photon excitation.

\begin{tabular}{l|c|c|c|c}
\hline \hline Excitation mode & $\begin{array}{c}\text { Excitation } \\
\text { wavelength }[\mathrm{nm}]\end{array}$ & $r_{0 \mathrm{i}}(\lambda)$ & $\Theta[\mathrm{ns}]$ & $\chi_{\mathrm{R}}^{2}$ \\
\hline One-photon $_{\text {global fit }}{ }^{\mathrm{y}}$ & 285 & 0.247 & 0.254 & 0.9 \\
& 280 & 0.222 & & \\
& 275 & 0.178 & & \\
& 264 & 0.131 & & \\
Three-photon & 260 & 0.112 & & \\
global fit & 780 & 0.119 & 0.251 & 1.0 \\
& 800 & 0.141 & & \\
& 825 & 0.192 & & \\
& 840 & 0.242 & & \\
\hline
\end{tabular}

${ }^{a}$ The correlation time $\Theta$ is a global parameter.

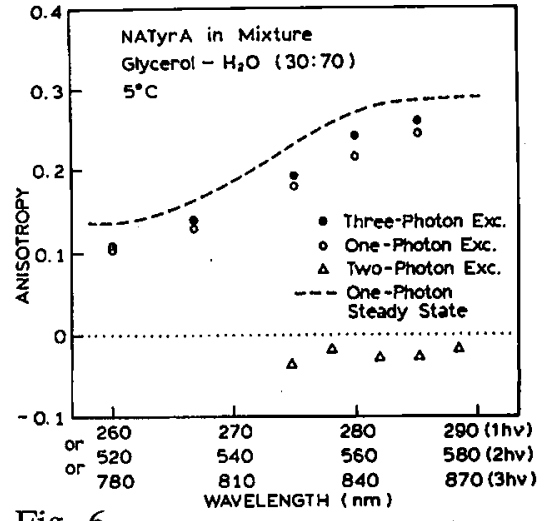

Fig. 6

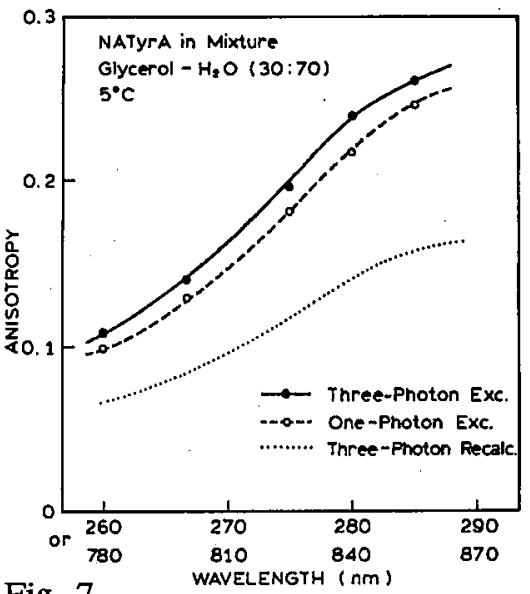

Fig. 7

Fig. 6. Excitation anisotropy spectrum for three-photon induced fluorescence of NATyrA ( $\bullet$ reconstructed from time-resolved measurements. Also shown are the one-photon values from time-resolved $(0)$ and steady state $(---)$ measurements. Also shown $(\Delta)$ are the fundamental anisotropies observed for two-photon excitation. Steady state measurements were performed in glycerol at $-60^{\circ} \mathrm{C}$.

Fig. 7. Comparison of the one $(O)$ and three-photon $(\bullet)$ excitation anisotropy spectra of NATyrA. Also shown are the three-photon anisotropies adjusted for $\cos ^{6} \theta$ to $\cos ^{2} \theta$ photoselection $(\cdots)$. 
The anisotropy spectrum of NATyrA with three-photon excitation is similar to that found with one-photon excitation (Fig. 6). However, the values for both one-photon and three-photon excitation are very different from that found previously for two-photon excitation (Fig. 6, triangles), which were near zero. These results show that it is not presently possible to predict the multi-photon anisotropies from the one-photon anisotropy data. It is important to remember that three-photon excitation results in anisotropy values which are 1.65-fold larger due to $\cos ^{6} \theta$ photoselection [40]. Hence we corrected the three-photon anisotropies for this effect. Our results indicate that the three-photon anisotropy of NATyrA is very similar to the one-photon values. Following this modification, our results indicate that the fundamental anisotropy of NATyrA for three photon-excitation is $40 \%$ lower than that found for one-photon excitation (Fig. 7). This can be seen by the three-photon values modified to one-photon photoselection.

We have made several general observations for multi-photon excitation versus one-photon excitation. To date, all emission spectra and intensity decay times have been the same for fluorophores excited by one, two or three photons. However, differences have been found in the anisotropies, in particular the time-zero or fundamental anisotropies. For many fluorophores the fundamental anisotropy for each mode of excitation can be explained as being due to differences in photoselection. This implies that the absorption and emission electronics transitions are essentially the same for each mode of excitation. However, we caution that a complete description of multi-photon transitions requires the use of tensors [41, 42]. For molecules like indole, tryptophan [43], and tyrosine [44] we found lower anisotropies with two-photon excitation than with one-photon excitation. This suggests that different electronic transitions are involved in multi-photon excitation of these molecules. Additional theory and experiments are needed to clarify the cause for this complex behavior.

\section{Engineered proteins as sensors}

During the past 10 years there have been extensive developments in the use of fluorescence for analytical and clinical chemistry [45-48]. Fluorophores are designed and synthesized to respond to the desired analyte, and to be specific for that analyte. If the spectral properties of the sensing fluorophore are appropriate, one can imagine the use of simple instruments based on laser diodes or light-emitting diodes for point-of-care testing (Fig. 8). Over the next decade one can predict the introduction of a variety of optical clinical devices based on measurements in syringes, capillary tubes, or array sensing chips.

While there have been substantial advances in the design of suitable fluorophores, it is often difficult to obtain the desired specificity and affinity for a particular application. In these cases one can rely on evolution and the wide range of specificities and affinities available from proteins. Because of advances in molecular biology and protein engineering it is possible to express or grow many proteins in bacterial systems. Additionally, the sequence of the proteins can be varied by site-directed mutagenesis to provide sites for labeling or to change the specificity or affinity of the protein for its substrate. In the following paragraphs we show how 


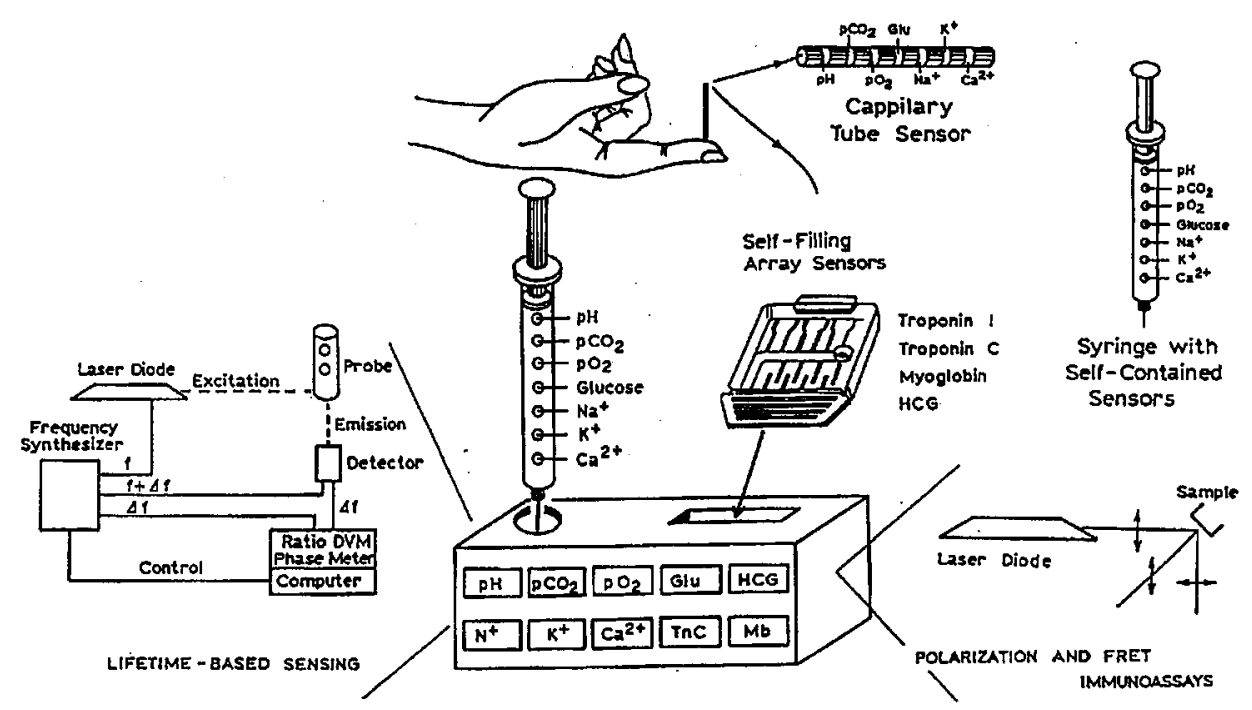

Fig. 8. Optical clinical chemistry. The sensing fluorophore may be placed on the walls of a syringe, in a self-filling capillary tube, or in an array sensor.

it was possible to develop a protein sensor specific for glucose with micromolar affinity based on mutagenesis of a bacterial protein [49].

Monitoring of blood glucose is central to the health care of diabetics. There are adverse long-term health consequences of elevated glucose, including cardiovascular disease and blindness. These adverse health effects have resulted in a worldwide effort to develop non-invasive methods to monitor blood glucose. A wide variety of methods have been proposed, including near infrared spectroscopy, optical rotation, amperometric, colorimetric and fluorescence detection. In spite of intensive efforts, no method is presently available for non-invasive measurement of blood glucose.

We now describe a new fluorescent method to measure glucose. The method is capable of measuring micromolar glucose concentrations without reagent consumption. This high sensitivity to glucose suggests the possibility of measuring the low glucose concentrations known to be present in extracted interstitial fluid [50]. Such samples are known to be painlessly available using methods which perturb the outermost layer of skin, the stratum corneum. These methods include laser ablation [51] and weak suction [52].

Our glucose sensor is based on the glucose/galactose binding protein (GGBP) from $E$. coli [53]. This protein binds glucose with a dissociation constant near $0.8 \mu \mathrm{M}[53,54]$. The single polypeptide chain of GGBP consists of two domains, the relative positions of which change upon binding of glucose [55] (Fig. 9). GGBP contains numerous surface lysine residues, and is thus not easily labeled at a specific location. A single cysteine mutant of GGBP was created by replacement of the amino acid residue at position 26 with a cysteine. This residue was labeled with the environmentally sensitive probe 2 -( $4^{\prime}$-iodoacetamidoanilino)naphthalene-6-sulfonic acid (I-ANS). 


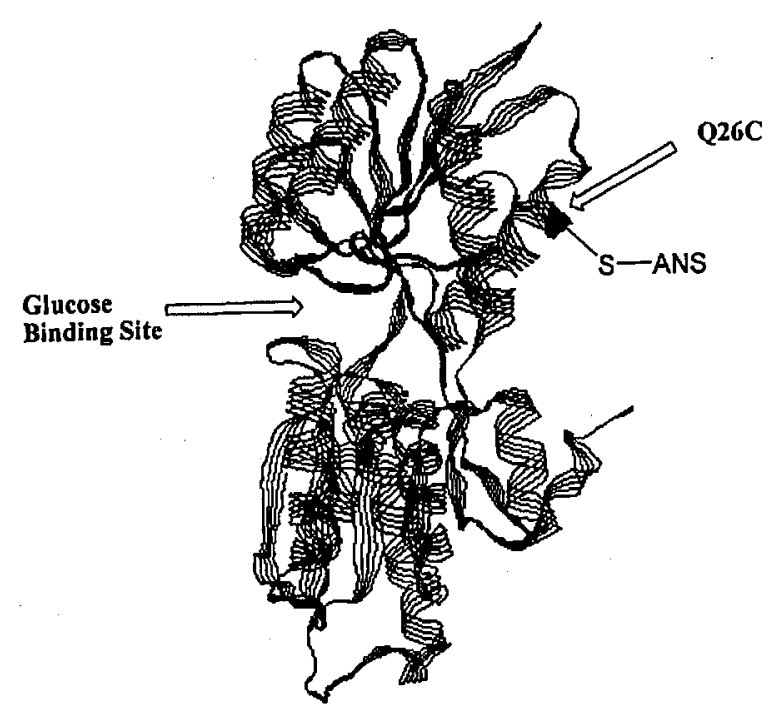

Fig. 9. Three-dimensional structure of the glucose/galactose binding protein showing the cysteine 26 labeled with ANS and the glucose binding site.

\subsection{Spectral properties of $A N S-G G B P$}

Emission spectra of ANS26-GGBP are shown in Fig. 10. These spectra are typical of the ANS fluorophore. Addition of micromolar concentrations of glucose resulted in an approximately 2 -fold decrease in the intensity of the ANS label. ANS is known to be sensitive to its local environment with lower intensities in more polar environments. The decrease in intensity suggests that ANS is displaced into the aqueous phase upon binding of glucose to ANS26-GGBP. This is consistent with the glucose-bound structure of GGBP (Fig. 9) where the residue on position 26 is pointing towards the aqueous phase. Similar results were obtained with other single cysteine mutants of GGBP [54]. In the absence of glucose, the GGBP is known to become more flexible [55]. It is likely that the flexibility of the protein in the absence of glucose allows ANS to position itself in a more hydrophobic surface of the protein resulting in increased fluorescence intensity.

Intensity decays of the ANS label were also measured (not shown). The intensity decays were found to be mostly insensitive to glucose. The absence of a useful change in lifetime was initially disappointing since one of our goals is a glucose sensor based on lifetime. Nonetheless, the spectra shown in Fig. 10 demonstrate the possibility of measuring micromolar glucose concentrations with this mutant of GGBP.

Because of the absence of a change in lifetime upon binding glucose, we designed a sensing strategy based on the modulation. The basic idea is to mix the protein, which displays a short nanosecond lifetime, with a long lifetime metal-ligand complex (MLC). At an appropriate intermediate light modulation frequency the modulation of the protein is near 1.0, and the modulation of the MLC is near zero. At this modulation frequency, the measured modulation represents the fraction of the emission due to the nanosecond fluorophore. If a sensor changes its intensity, 


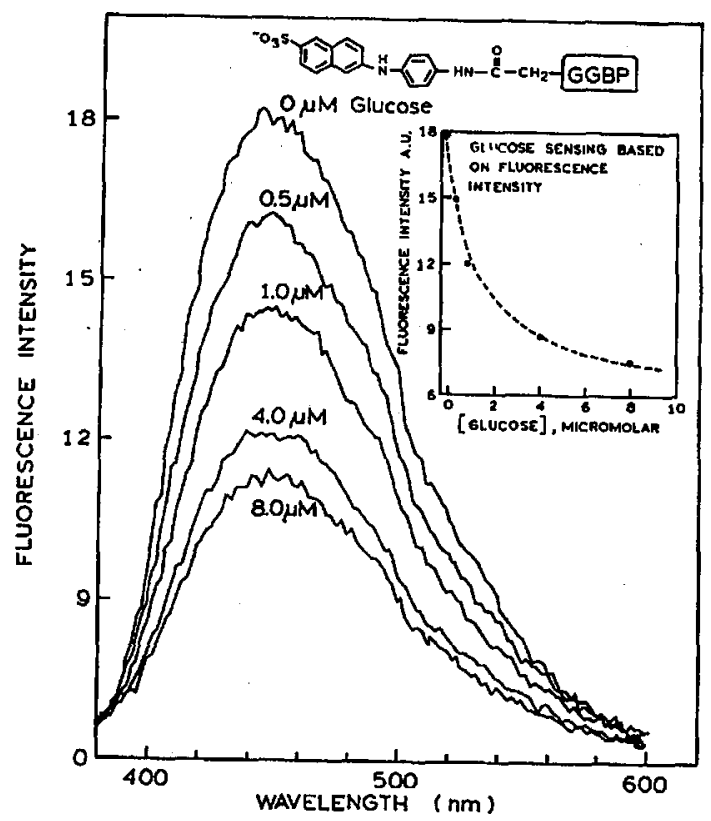

Fig. 10. Emission spectra (intensity in arbitrary units) of ANS26-GGBP in the presence of 0 to $8 \mu \mathrm{M}$ glucose. [GGBP] $=0.25 \mu \mathrm{M}$, excitation at $325 \mathrm{~nm}$. The inset shows the change in intensity versus glucose concentration.

then the low frequency modulation is also changed to represent the fraction of the total emission from the sensor. We used this concept and the intensity changes found for ANS26-GGBP (Fig. 10) to develop a low frequency modulation sensor for glucose.

Prior to examining the glucose data it is informative to simulate the expected results. Simulated frequency-domain data for a mixture of fluorophores are shown in Fig. 11. The lifetimes were assumed to be $\tau_{1}=5 \mathrm{~ns}$ and $\tau_{2}=1000 \mathrm{~ns}=1 \mu \mathrm{s}$. The lifetime of $5 \mathrm{~ns}$ is comparable to the mean lifetime of ANS26-GGBP. Metal-ligand Re complexes with lifetimes of over $1 \mu$ s are now available [56, 57]. For these simulations we assumed the fractional intensity of the $5 \mathrm{~ns}$ component changed from 0.1 to 0.76 . There is a region near $2 \mathrm{MHz}$ where the modulation is almost independent of modulation frequency. Importantly, the modulation is sensitive to the fractional intensity of the short-lived component. For the assumed lifetimes the modulation at $2 \mathrm{MHz}$ is essentially equal to the amplitude of the short-lived component.

We used this concept to measure glucose. The labeled protein ANS26-GGBP was placed adjacent to a ruthenium complex to result in a fractional GGBP intensity near 0.87 in the absence of glucose. The Ru complex was in a thin film outside the cuvette containing ANS26-GGBP. Frequency responses are shown in Fig. 12. Importantly, the modulation at $2.1 \mathrm{MHz}$ decreases in the presence of glucose, as expected for the decreased intensity from ANS26-GGBP. These changes in modulation were used to prepare a calibration curve for glucose (Fig. 13). These data demonstrate that the ANS26-GGBP can be used to quantify micromolar concen- 


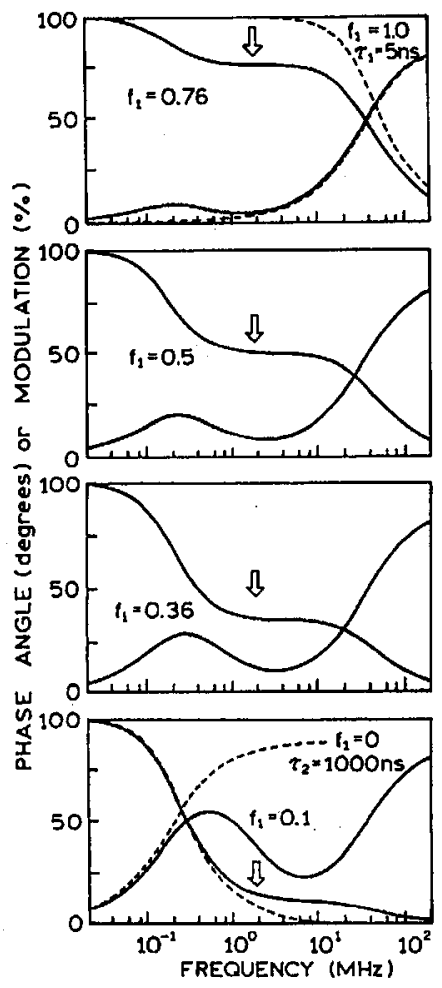

Fig. 11. Simulated frequency-domain intensity decays for a mixture of fluorophores, $\tau_{1}=5 \mathrm{~ns}, \tau_{2}=1000 \mathrm{~ns}, f_{1}=0.76$ to 0.1 .

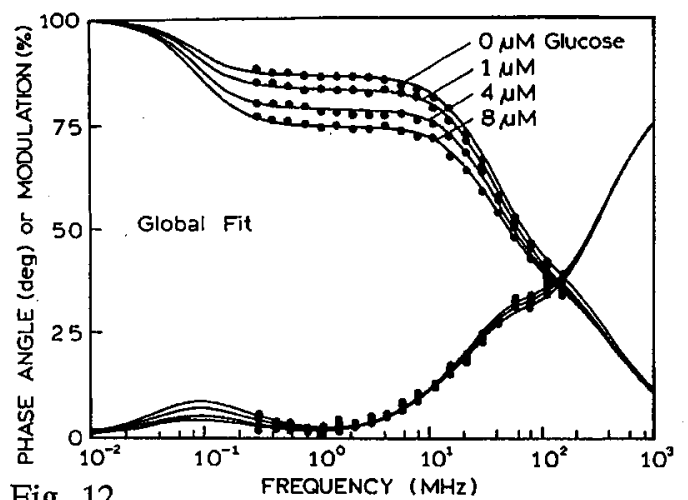

Fig. 12

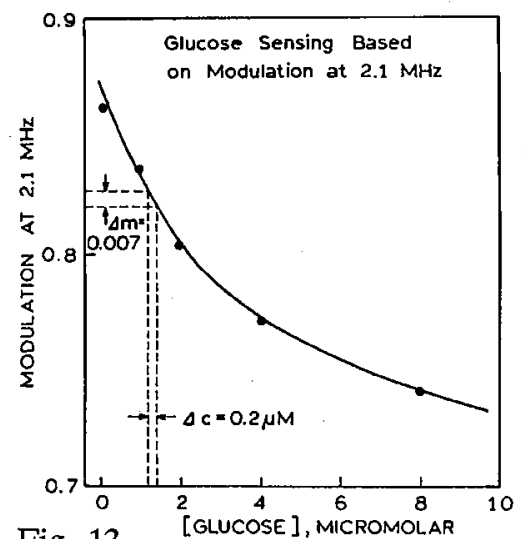

Fig. 13

Fig. 12. Frequency responses of the glucose sensor at $0,1,4$, and $8 \mu \mathrm{M}$ glucose.

Fig. 13. Effect of glucose on modulation of the glucose sensor at $2.1 \mathrm{MHz}$.

trations of glucose. Modulation measurements accurate to $\Delta m= \pm 0.007$ would result in glucose concentrations accurate to $\Delta c= \pm 0.2 \mu \mathrm{M}$. We expect future labeled GGBP mutants will display larger changes in fluorescence and yield more accurate glucose measurements. 
It is interesting to consider the opto-electronics required for modulation-based sensing. It is now known that blue light emitting diodes (LEDs) can be amplitude modulated from 0.1 to $100 \mathrm{MHz}$ [58]. Also, LEDs with ultraviolet output near $380 \mathrm{~nm}$ are available and can be modulated to $100 \mathrm{MHz}$ [59]. Electroluminescent devices can also be modulated at $\mathrm{MHz}$ frequencies [60]. Recent experiments show the feasibility of constructing low-cost instrumentation for phase-modulation measurements up to $100 \mathrm{MHz}$ [61]. Hence simple inexpensive light sources could be used for a modulation glucose sensor.

A device for modulation-based sensing can be simpler than the usual phase-modulation instruments. For phase angle measurements the detector must be modulated with a fixed phase relationship to the modulated excitation. Modulation measurements can be performed without the phase-locked relationship, simplifying the electronics. These considerations suggest that a portable battery powered device can be designed to monitor glucose. This device could be used with diluted blood. The sensitivity to low glucose concentrations suggests its use to monitor glucose in extracted interstitial fluid.

We note that these results on the glucose binding protein were not presented during my lecture at the Jabłoński Conference. However, we felt that the proceeding volume should contain the most recent developments in sensing.

\section{Microsecond biopolymer dynamics}

Hundreds of fluorophores are presently a vailable, as described in the scientific literature [61] or from commercial sources [62]. In spite of this plethora of compounds, there is little diversity in the fluorescence decay times. The vast majority of fluorophores display lifetimes from 1 to $10 \mathrm{~ns}$. In general, the dynamic information content of a fluorescent experiment is limited to three times comparable to the lifetime. Hence, fluorescence typically provides information on the nanosecond timescale. While this timescale is useful for studies of the internal dynamics of proteins and membranes, longer timescales are needed to study macromolecular dynamics and association-dissociation phenomena. From this perspective fluorescence has been trapped on the nanosecond timescale, with limited information available at longer times.

In this laboratory we have developed a new class of luminescent probes which have microsecond decay times. These probes are transition metal-ligand complexes which contain organic ligands. A wide variety of such compounds have been synthesized and characterized for possible use in solar energy conversion. However, these luminescent complexes have not been used as probes. A valuable characteristic of fluorescent probes is polarized emission when excited with polarized light. This property provides information about rotational motions from the steady-state or time-resolved anisotropies. We found that the ruthenium complexes display polarized emission, allowing their use as anisotropy probes [63-67]. For these complexes the emission is from a metal-ligand charge transfer (MLCT) state. We also developed rhenium (Re) complexes which display higher quantum yields and longer lifetimes than the ruthenium $(\mathrm{Ru})$ complexes [68-70]. We now summarize recent results for a Re-lipid conjugate as a probe of membrane dynamics. 


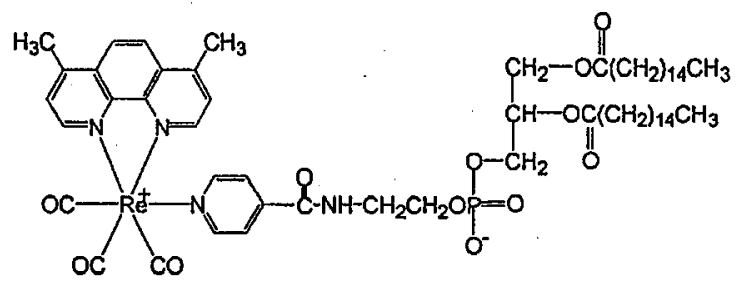

Fig. 14. Molecular structure of the phospholipid analogue of $\left[\operatorname{Re}\left(4,7-\mathrm{Me}_{2}\right.\right.$ phen $)$ $\left.(\mathrm{CO})_{3}(4-\mathrm{COOHPy})\right]^{+}(\mathrm{Re}-\mathrm{PE})$.

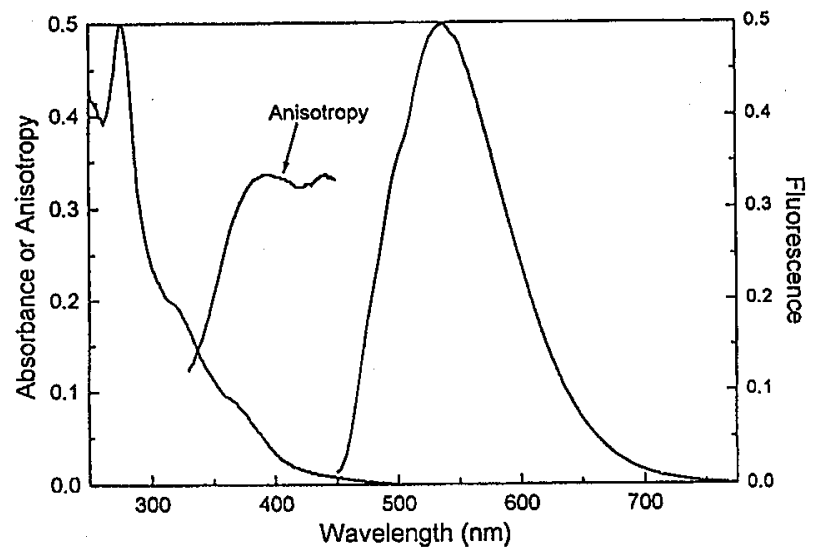

Fig. 15. Absorption and emission spectra of Re-PE in DPPG vesicles at $20^{\circ} \mathrm{C}$. Also shown is the excitation anisotropy spectrum of the parent compound $\left[\operatorname{Re}\left(4,7-\mathrm{Me}_{2} \text { phen }\right)(\mathrm{CO})_{3}(4-\mathrm{COOHPy})\right]^{+}$in glycerol-methanol at $-60^{\circ} \mathrm{C}$.

Our rhenium-lipid probe (Re-PE) is shown in Fig. 14, which was synthesized as described in [70]. The absorption and emission spectra of Re-PE in DPPG vesicles at $20^{\circ} \mathrm{C}$ are shown in Fig. 15. The strong UV band at $280 \mathrm{~nm}$ and the broad absorbance above $300 \mathrm{~nm}$ are due to the ligand-centered and MLCT transitions, respectively. Emission of Re-PE is broad with the maximum peak at $535 \mathrm{~nm}$, which has been assigned as MLCT-based luminescence [71]. These two spectra display a large Stokes' shift. Scattered light from membrane suspensions can be easily eliminated from the detected emission. A large Stokes' shift is also useful because the labeled membranes can contain relatively large amounts of probe without loss of anisotropy due to energy migration [72].

Frequency-domain intensity decays of Re-PE in lipid membranes are shown in Fig. 16. These decays are best fit to three exponential decay law and the recovered lifetimes are listed in Table II. The mean lifetimes, calculated using $\bar{\tau}=\sum_{i} \alpha_{i} \tau_{i}^{2} / \sum_{i} \alpha_{i} \tau_{i}$, are approximately unchanged for Re-PE in DPPG vesicles at 10 and $30^{\circ} \mathrm{C}$, below the gel phase to fluid phase transition temperature at $41^{\circ} \mathrm{C}$. However, the mean lifetime above $41^{\circ} \mathrm{C}$ is substantially shorter. The result is likely due to changes in the probe environment which occur during the phase transition of the lipid vesicles. The fact that the intensity of Re-PE is influenced by the lipid is seen by noting the single exponential decay of $5.9 \mu$ s in deaerated chloroform (not shown). 


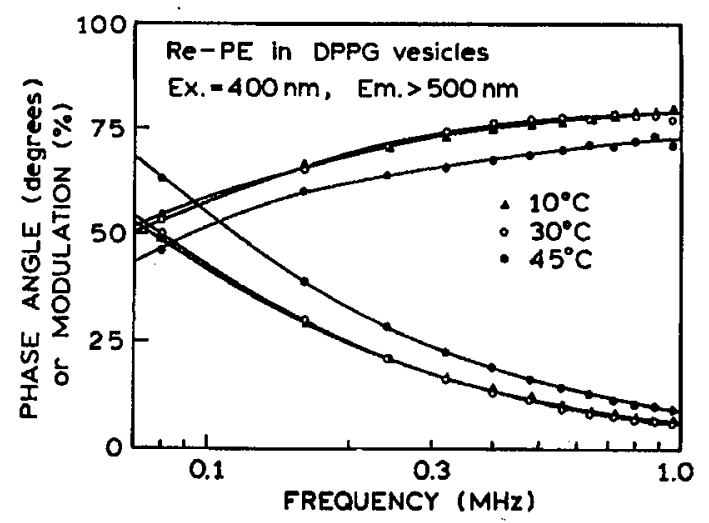

Fig. 16. Frequency-domain intensity decays of Re-PE incorporated in DPPG vesicles at various temperatures.

TABLE II

Frequency-domain intensity and anisotropy decays of Re-PE in DPPG vesicles at various temperatures.

\begin{tabular}{c|c|c|c|c|c|c|c}
\hline \hline \multicolumn{5}{c|}{ Intensity decays } & \multicolumn{3}{c}{ Anisotropy decays } \\
\hline$T\left[{ }^{\circ} \mathrm{C}\right]$ & $\chi_{\mathrm{R}}^{2}$ & $\alpha_{i}$ & $\tau_{i}[\mathrm{~ns}]$ & $\bar{\tau}[\mathrm{ns}]$ & $\theta_{i}[\mathrm{~ns}]$ & $r_{0} g_{i}$ & $\chi_{\mathrm{R}}^{2}$ \\
\hline 10 & & 0.238 & 112.6 & 3791.1 & 30.3 & 0.298 & \\
& & 0.406 & 1142.9 & & 1269.8 & 0.032 & $1.4(35.7)^{a}$ \\
& \multirow{4}{*}{$1.8(130.6)^{a}$} & 0.356 & 4676.3 & & & & \\
& & 0.441 & 185.0 & 3845.4 & 14.0 & 0.301 & \\
& & 0.292 & 1096.0 & & 1305.1 & 0.029 & $3.2(31.2)$ \\
45 & $2.4(490.6)$ & 0.267 & 4772.0 & & & & \\
& & 0.476 & 23.8 & 2340.0 & 10.3 & 0.315 & \\
& & 0.189 & 353.7 & & 2431.1 & 0.015 & $2.9(14.0)$ \\
& $1.5(341.1)$ & 0.335 & 2727.7 & & & & \\
\hline
\end{tabular}

${ }^{a}$ In parentheses are $\chi_{\mathrm{R}}^{2}$ values for the best single exponential fits.

For use as an anisotropy probe to study the hydrodynamics of lipid membranes, the metal-ligand complex must display polarized emission. We examined the excitation anisotropy spectrum of the parent compound, $\left[\operatorname{Re}\left(4,7-\mathrm{Me}_{2}\right.\right.$ phen $)$ $\left.(\mathrm{CO})_{3}(4-\mathrm{COOHPy})\right]\left(\mathrm{PF}_{6}\right)$, dissolved in glycerol-methanol $(95: 5, \mathrm{~V}: \mathrm{V})$ under vitrified condition $\left(-60^{\circ} \mathrm{C}\right)$ to avoid rotational diffusion (Fig. 15). This complex shows a maximal anisotropy of 0.33 , which is easily sufficient for measurement of the time-resolved anisotropies. In other studies we have found $[73,74]$ that all the rhenium complexes we examined displayed highly polarized emission. It seems that the presence of a single chromophoric group, the diimine ligand, insures a defined direction for the electronic transition and a high fundamental anisotropy.

The frequency-domain anisotropy decays of DPPG vesicles labeled with Re-PE at various temperatures are shown in Fig. 17. The excitation wavelength 

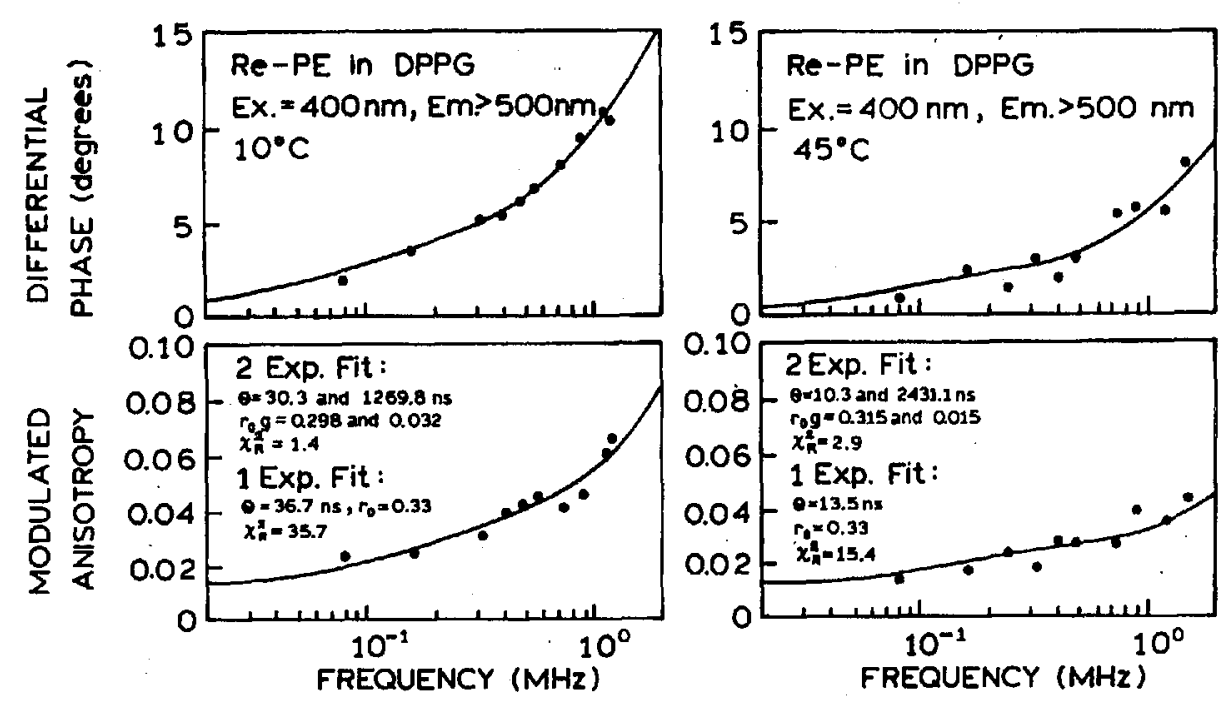

Fig. 17. Frequency-domain anisotropy decays of DPPG vesicles labeled with Re-PE.

was $400 \mathrm{~nm}$ at which the fundamental anisotropy of Re-PE is near 0.33 . Analysis of these data in terms of two rotational correlation times using Eq. (2) are summarized in Table II. The total anisotropy was fixed to 0.33 during the analyses. The short rotational correlation time near $10-30$ ns probably reflects the fast rotational motion of rhenium lipid by itself. The longer correlation time near $1.3 \mu \mathrm{s}$ is consistent with that expected for overall rotational diffusion of phospholipid vesicles with the diameter between 200 and $250 \AA$. According to the Stokes-Einstein equation the rotational correlation time is given by $\theta=\eta V / R T$, where $\eta$ is the viscosity of the medium and is equal to $1 \mathrm{cP}, V$ is the volume of the lipid vesicles, and $T=297 \mathrm{~K}$. Vesicles with diameters of 200 and $250 \AA$ are expected $[65,66]$ to display correlation times of 1034 and 2020 ns, respectively, which are comparable to the recovered values. The amplitude of the long correlation time decreased by half as the temperature was increased from 10 to $45^{\circ} \mathrm{C}$. However, the slow vesicular rotational diffusion persists even at $45^{\circ} \mathrm{C}$, above the lipid phase transition temperature $\left(41^{\circ} \mathrm{C}\right)$. Also, the correlation time recovered at $45^{\circ} \mathrm{C}$ is as long or longer than those found below the phase transition temperature, possibly resulting from the low value of the fractional anisotropy contributed by this slow rotational motion which affect the accuracy of the analysis. However, it is also possible that the long correlation time has increased due to aggregation of the vesicles.

It is interesting to examine the time-dependent anisotropy decays reconstructed from the recovered parameters (Fig. 18). At temperatures above and below the phase transition temperature of the lipid the anisotropies show rapid decreases below $100 \mathrm{~ns}$, followed by a much slower loss of the remaining anisotropy over several microseconds. For use as an anisotropy probe it is important to know if the complex anisotropy decay displayed by the MLC probe is due to its motions, and not due to some fundamental property of the probe. Hence we examined the intensity and anisotropy decay of the parent component $\operatorname{Re}\left(4,7-\mathrm{Me}_{2}\right.$ phen $)(\mathrm{CO})_{3}$ 

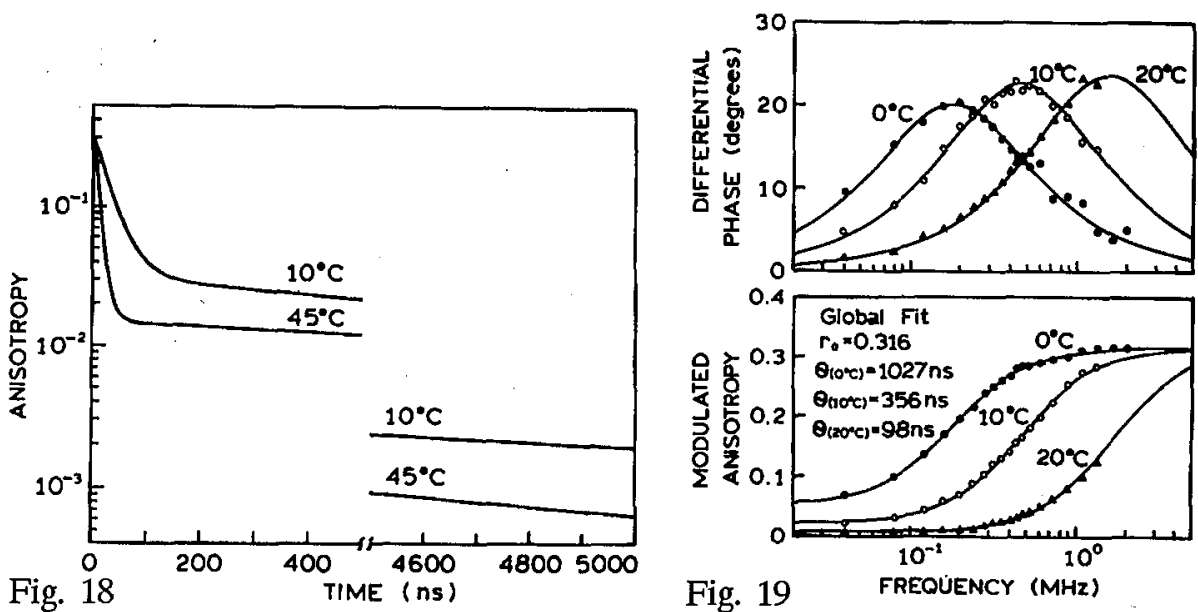

Fig. 19

Fig. 18. Reconstructed time-domain anisotropy decays of DPPG vesicles labeled with Re-PE.

Fig. 19 Frequency-domain anisotropy decay of $\operatorname{Re}\left(\mathrm{Me}_{2}\right.$-phen $)(\mathrm{CO})_{3}(\mathrm{COOHPy}) \mathrm{PF}_{6}$ in glycerol.

TABLE III

Fluorescence intensity and anisotropy decay analyses of ( $\mathrm{Re}\left(4,7\right.$-dimethyl-phen $\left.(\mathrm{CO})_{3} \mathrm{py}-\mathrm{COOH}\right) \mathrm{PF}_{6}$ in glycerol at various temperatures.

\begin{tabular}{l|c|c|c|c|c|c|c}
\hline \hline$T\left[{ }^{\circ} \mathrm{C}\right]$ & $\tau[\mathrm{ns}]$ & $\chi_{\mathrm{R}}^{2}$ & $\begin{array}{c}\chi_{\mathrm{R}}^{2}(1 \exp ) / \\
\chi_{\mathrm{R}}^{2}(2 \exp )\end{array}$ & $r_{0}$ & $\Theta[\mathrm{ns}]$ & $\chi_{\mathrm{R}}^{2}$ & $\begin{array}{c}\chi_{\mathrm{R}}^{2}(1 \exp ) / \\
\chi_{\mathrm{R}}^{2}(2 \exp )\end{array}$ \\
\hline 0 & 5101.0 & 2.4 & 1.01 & 0.316 & 1026.7 & 3.7 & 1.07 \\
10 & 4752.6 & 2.3 & 1.06 & 0.316 & 356.5 & 2.3 & 1.04 \\
20 & 4316.8 & 3.4 & 1.08 & 0.314 & 98.4 & 0.9 & 1.00 \\
Global fit & & & & & & & \\
0 & - & & & 0.316 & 1027.4 & 2.3 & 1.03 \\
10 & - & & & & 356.1 & & \\
20 & - & & & & 97.6 & &
\end{tabular}

(4-COOHPy) in glycerol. The intensity decays were found to be single exponential at all studied temperatures (Fig. 19). Importantly, the anisotropy decays displayed a single correlation time and a time-zero anisotropy near 0.32 , with no evidence of a more rapid component (Table III). Hence the probe does not display any unusual anisotropy behavior, and can be reliably expected to report on microsecond molecular motions.

In order to obtain additional information on the overall hydrodynamics of membranes it would be valuable to decrease the amplitude of the faster motion. This could possibly be accomplished by attaching the rhenium complex to two 
lipid molecules, as has been done previously for a luminescent ruthenium complex $[65,66]$. In summary, long lifetime complexes are promising new probes for the microsecond dynamics of biological macromolecules and their assemblies.

\section{Perspectives on fluorescence}

$\dot{W e}$ are presently experiencing an era of vigorous growth in the principles and applications of fluorescence. The uses expand on a daily basis as fluorescence detection completes its progressive replacement of radioactivity as the basis of medical testing. The human genome project would not be possible, let alone be a competition between private companies and federally-funded laboratories, without the rapid and inexpensive detection based on labeled nucleotides. It is now possible to directly grow fluorescent proteins based on fusion with the gene for green fluorescent protein (GFP). Fluorescent probes are becoming increasingly specific, and will become even more specific with the introduction of engineered proteins as sensors. Advances in optics, electronics, and micro-machines are all contributing to increased sensitivity with decreasing costs and complexity. It is remarkable that the basic investigations of A. Jabłoński, G. Weber and other pioneers have evolved into the versatile and robust technology we call fluorescence spectroscopy.

\section{Acknowledgment}

The authors acknowledge the National Institutes of Health RR-10955 to Dr. Govind Rao and GM-35154, RR-08119, and RR10416-01 to Joseph R. Lakowicz for the support of this research.

\section{References}

[1] M. Göppert-Mayer, Ann. Phys. 9, 73 (1931).

[2] D.M. Friedrich, W.M. McClain, Annu. Rev. Phys. Chem. 31, 559 (1980).

[3] M.J. Wirth, A. Koskelo, M.J. Sanders, Appl. Spectrosc. 35, 14 (1981).

[4] W.L. Peticolas, Annu. Rev. Phys. Chem. 18, 233 (1967).

[5] R.D. Jones, P.R. Callis, Chem. Phys. Lett. 144, 58 (1989).

[6] M.J. Wirth, A.C. Koskelo, C.E. Mohler, B.L. Lentz, Anal. Chem. 53, 2045 (1981).

[7] M.B. Masthay, L.A. Findsne, B.M. Pierce, D.F. Bocian, J.S. Lindsey, R.R. Birge, J. Chem. Phys. 84, 3901 (1986).

[8] H.L.-B. Fang, R.J. Thrash, G.E. Leroi, Chem. Phys. Lett. 57, 59 (1978).

[9] B. Hudson, Ann. Rev. Phys. Chem. 25, 437 (1974).

[10] R.R. Birge, Acc. Chem. Res. 19, 138 (1986).

[11] B.E. Anderson, R.D. Jones, A.A. Rehms, P. Ilich, P.R. Callis, Chem. Phys. Lett. 125, 106 (1986).

[12] A. Rehms, P.R. Callis, Chem. Phys. Lett. 140, 83 (1987).

[13] W.D. Pfeffer, E.S. Yeung, Anal. Chem. 58, 2103 (1986).

[14] M.J. Wirth, H.O. Fatunmbi, Anal. Chem. 62, 973 (1990).

[15] W.M. McClain, J. Chem. Phys. 55, 2789 (1971).

[16] J.R. Lakowicz, I. Gryczynski, J. Kusba, E. Danielsen, J. Fluoresc. 2, 247 (1992). 
[17] J.R. Lakowicz, I. Gryczynski, J. Fluoresc. 2, 117 (1992).

[18] I. Gryczynski, J.R. Lakowicz, J. Fluoresc. 4, 331 (1994).

[19] J.R. Lakowicz, I. Gryczynski, Biophys. Chem. 45, 1 (1993).

[20] J.R. Lakowicz, I. Gryczynski, Biophys. Chem. 47, 1 (1993).

[21] A. Kawski, I. Gryczynski, Z. Gryczynski, Z. Naturforsch. 481, 551 (1993).

[22] J.R. Lakowicz, I. Gryczynski, Z. Gryczynski, E. Danielsen, M.J. Wirth, J. Phys. Chem. 98, 3000 (1992).

[23] J.R. Lakowicz, I. Gryczynski, K. Nowaczyk, Spectrochim. Acta A 53, 1637 (1997).

[24] J.R. Lakowicz, I. Gryczynski, Biospectroscopy 1, 3 (1995).

[25] I. Gryczynski, J.R. Lakowicz, Photochem. Photobiol. 62, 426 (1995).

[26] I. Gryczynski, A. Razynska, J.R. Lakowicz, Biophys. Chem. 57, 291 (1996).

[27] R.A. Agbaria, I. Gryczynski, H. Malak, J.R. Lakowicz, Biospectroscopy 2, 213 (1996).

[28] W. Denk, J.H. Strickler, W.W. Webb, Science 248, 73 (1990).

[29] D.W. Piston, D.R. Sandison, W.W. Webb, Proc. SPIE 1640, 379 (1992).

[30] S.W. Hell, S. Lindek, E.H.K. Stelzer, J. Mod. Opt. 41, 675 (1994).

[31] S.W. Hell, M. Schrader, P.E. Hanninen, E. Soini, Opt. Commun. 120, 129 (1995).

[32] I. Gryczynski, H. Malak, S.W. Hell, J.R. Lakowicz, J. Biomed. Optics 1, 473 (1996).

[33] I. Gryczynski, H. Malak, J.R. Lakowicz, Biospectroscopy 2, 9 (1996).

[34] I. Gryczynski, H. Malak, J.R. Lakowicz, H.C. Cheung, J. Robinson, P.K. Umeda, Biophys. J. 71, 3448 (1996).

[35] J.R. Lakowicz, I. Gryczynski, H. Malak, M. Schrader, P. Engelhardt, H. Kano, S.W. Hell, Biophys. J. 72, 567 (1997).

[36] H. Malak, I. Gryczynski, J.D. Dattelbaum, J.R. Lakowicz, J. Fluoresc. 77, 99 (1977).

[37] S.W. Hell, S.K. Bahlmann, M. Schrader, A. Soni, H. Malak, I. Gryczynski, J.R. Lakowicz, J. Biomed. Optics 1, 71 (1996).

[38] I. Gryczynski, H. Malak, J.R. Lakowicz, Biophys. Chem., 1998, submitted.

[39] J.R. Lakowicz, G. Laczko, I. Gryczynski, Biochemistry 26, 82 (1987).

[40] I. Gryczynski, H. Malak, J.R. Lakowicz, Chem. Phys. Lett. 245, 30 (1995).

[41] P.R. Callis, J. Chem. Phys. 99, 27 (1993).

[42] S.-Y. Chen, B.W. Van Der Meer, Biophys. J. 64, 1567 (1993).

[43] J.R. Lakowicz, I. Gryczynski, E. Danielsen, J.K. Frisoli, Chem. Phys. Lett. 194, 282 (1992).

[44] B. Kierdaszuk, I. Gryczynski, A. Modrak-Wojcik, A. Bzowska, D. Shugar, J.R. Lakowicz, Photochem. Photobiol. 61, 319 (1995).

[45] Advances in Fluorescence Sensing Technology III, Ed. R.B. Thompson, Vol. 2980, SPIE Proc., San Jose 1997, p. 582.

[46] Fiber Optic Chemical Sensors and Biosensors, Ed. O.S. Wolfbeis, CRC Press, Boca Raton 1991, Ch. II, p. 267.

[47] Topics in Fluorescence Spectroscopy: Probe Design and Chemical Sensing, Ed. J.R. Lakowicz, Plenum Press, New York 1994, p. 501. 
[48] Proc. 3rd European Conf. on Optical Chemical Sensors and Biosensors, Part I - Plenary and Parallel Sessions, Part II - Poster Sessions, Europt(R)ode III, Sensors and Actuators B, Vol. 38, Ed. R.E. Kunz, Elsevier, Amsterdam 1997.

[49] L. Tolosa, I. Gryczynski, L. Eichhorn, J.D. Dattelbaum, F.N. Castellano, J.R. Lakowicz, Anal. Biochem., submitted.

[50] J.A. Tamada, N.J.V. Bohannon, R.O. Potts, Nature Med. 1, 1198 (1995).

[51] S.L. Jacques, D.J. McAuliffe, I.H. Blank, J.A. Parrish, J. Investigative Dermatology 88, 88 (1987).

[52] N. Ito, S. Kayashima, J. Kimura, T. Kuriyama, T. Arai, M. Kikuchi, N. Nagata, Biosensors 32, 242 (1994).

[53] W. Boos, A.S. Gordon, R.E. Hall, H.D. Price, J. Biol. Chem. 247, 917 (1972).

[54] J.S. Marvin, H.W. Hellinga, J. Am. Chem. Soc. 120, 7 (1998).

[55] C.L. Careaga, J. Sutherland, J. Sabeti, J.J. Falke, Biochemistry 34, 3048 (1995).

[56] X.Q. Guo, F.N. Castellano, L. Li, H. Szmacinski, J.R. Lakowicz, J. Sipior, Anal. Biochem. 254, 179 (1997).

[57] X.Q. Guo, F.N. Castellano, L. Li, J.R. Lakowicz, Anal. Chem. 70, 632 (1998).

[58] J. Sipior, G.M. Carter, J.R. Lakowicz, G. Rao, Rev. Sci. Instrum. 67, 3795 (1996).

[59] J. Sipior, G.M. Carter, J.R. Lakowicz, G. Rao, Rev. Sci. Instrum. 68, 2666 (1997).

[60] K.W. Berndt, J.R. Lakowicz, Anal. Biochem. 201, 319 (1992).

[61] P. Harms, J. Sipior, N. Ram, G. Carter, G. Rao, Anal. Chem., 1999, in preparation.

[62] Molecular Probes Catalogue, 6th ed., Ed. R.P. Haugland, Eugene, Oregon 1996.

[63] E. Terpetschnig, H. Szmacinski, H. Malak, J.R. Lakowicz, Biophys. J. 68, 342 (1995).

[64] H. Szmacinski, E. Terpetschnig, J.R. Lakowicz, Biophys. Chem. 62, 109 (1996).

[65] L. Li, H. Szmacinski, J.R. Lakowicz, Biospectroscopy 3, 155 (1997).

[66] L. Li, H. Szmacinski, J.R. Lakowicz, Anal. Biochem. 244, 80 (1997).

[67] F.N. Castellano, J.D. Dattelbaum, J.R. Lakowicz, Anal. Biochem. 255, 165 (1998).

[68] X.Q. Guo, F.N. Castellano, L. Li, H. Szmacinski, J.R. Lakowicz, J. Sipior, Anal. Biochem. 254, 179 (1997).

[69] X.Q. Guo, F.N. Castellano, L. Li, J.R. Lakowicz, Anal. Chem. 70, 632 (1998).

[70] L. Li, F.N. Castellano, I. Gryczynski, J.R. Lakowicz, Chem. Phys. Lipids, submitted.

[71] L. Wallace, D.P. Rillema, Inorg. Chem. 32, 3836 (1993).

[72] J.R. Lakowicz, Principles of Fluorescence Spectroscopy, 2nd ed., Plenum Press, New York, in press (1999).

[73] J.R. Lakowicz, Z. Murtaza, W.E. Jones, K. Kim, H. Szmacinski, J. Fluoresc. 6, 245 (1996).

[74] F.N. Castellano, J.D. Dattelbaum, J.R. Lakowicz, manuscript in preparation. 